\title{
Can pre-operative carbohydrate loading be used in diabetic patients undergoing colorectal surgery?
}

\author{
AFFIFA FARRUKH, KATH HIGGINS, BALJIT SINGH, ROBERT GREGORY
}

\begin{abstract}
The introduction of enhanced recovery after surgery (ERAS) has been associated with shortening post-operative recovery. It achieves such outcomes by minimizing the physical and physiological trauma of surgery. Benefits include superior pain control, reduced duration of ileus, improved pulmonary function and a reduction in thromboembolic and cardiac events. Within the ERAS approach the role of oral carbohydrate supplements is based on dealing with insulin resistance which characterizes periods of stress. Aggressive control of blood glucose levels has been shown to benefit both diabetic and non-diabetic patients admitted to intensive care units, however original studies in this area have not been consistently reproducible. The development of low osmolality carbohydrate drinks during the mid 1990s opened up the possibility of extending these benefits to surgical patients by providing them with a carbohydrate load two to three hours prior to anaesthesia. The benefits of the ERAS approach to colorectal surgery has been confirmed in several reports. However, its role in diabetic patients has, as yet, received limited attention. This review examines this limited number of publications and considers the potential benefit of preoperative carbohydrate loading in all diabetic patients.

Br J Diabetes Vasc Dis 2014;14:102-104
\end{abstract}

Key words: diabetes, surgery, carbohydrate loading, pre-operative

\section{Introduction}

The introduction of ERAS has been associated with shortening post-operative recovery. It achieves such outcomes by minimizing the physical and physiological trauma of surgery. It is claimed

Departments of Digestive Diseases and Diabetes, University Hospitals of Leicester NHS Trust, Leicester, UK

Address for correspondence: Dr Affifa Farrukh

Department of Digestive Diseases, Leicester General Hospital,

Gwendolen Road, Leicester, LE5 4PW, UK

Tel: +44 (0)1162 584787

E-mail: farrukh_affi@yahoo.com

http://dx.doi.org/10.15277/bjdvd.2014.024

\section{Abbreviations and acronyms}

ERAS

enhanced recovery after surgery

that such an approach will benefit all patients. ${ }^{1}$ The overall strategy requires that patients are given partnership and responsibility for their care, health is optimized prior to surgery and care and rehabilitation are evidence based. Such an approach has called into question the traditional pre-operative overnight fast and the use of bowel preparation for colonic surgery amongst other aspects of care. The benefit of such an approach for patients undergoing colorectal surgery is recognized throughout the world. 1,2 Benefits include superior pain control, reduced duration of ileus, improved pulmonary function and a reduction in thrombo-embolic and cardiac events. Within the ERAS approach the role of oral carbohydrate supplements is based on dealing with insulin resistance which characterizes periods of stress. It improves post-operative glycaemic control through endogenous insulin release, not only by reducing the risk of hyperglycaemia during post-operative nutrition but also by improving nitrogen economy and therefore maintaining muscle strength. ${ }^{3}$

\section{Historic background}

In 1877 Bernard demonstrated that stress disturbed glucose homeostasis when he reported hyperglycaemia in association with haemorrhage. ${ }^{4}$ This was the first recognition of the existence of insulin resistance. Although this physiological response in which glucose reserves are directed to non-insulin dependent tissues, such as the brain, may be beneficial after trauma, Ljungqvist et al. questioned whether this was true following surgery. ${ }^{5}$ These doubts arose following work on insulin resistance as a marker of surgical stress. ${ }^{6}$ Elevated blood glucose level is associated with an increased incidence of infections following surgery. ${ }^{7}$ Improved glycaemic control was associated with fewer deep sternal infections in diabetic patients who underwent cardiac surgery. ${ }^{8}$ Aggressive control of blood glucose levels has been shown to be of benefit to both diabetic and non-diabetic patients admitted to an intensive care unit, with significant reductions in death rates from multiple organ dysfunction. ${ }^{9}$ However, since this study was published in 2001, these results have been difficult to reproduce and the NICE-SUGAR study ${ }^{10}$ and a metaanalysis 11 have informed consensus opinion that "aggressive" blood glucose control is potentially harmful. The main issue is that an "aggressive" approach to glycaemic control increases 
the likelihood of hypoglycaemic episodes. For this reason moderate control is associated with lower mortality amongst diabetic patients, although this is probably not true for people without diabetes. ${ }^{12}$ Indeed a recent study of non-diabetic patients undergoing hepato-biliary surgery again demonstrated the benefits of aggressive control of blood sugar levels leading to reduced incidence of infections and shorter hospital stays, and none of the patients in the study had any episodes of hypoglycaemia. ${ }^{13}$ Clearly the need to ensure that blood glucose is kept within an acceptable range is crucial to the success of any intensive insulin regime. ${ }^{14}$ Against this background, Ljungqvist et al. ${ }^{5}$ interpreted their own and other work as showing the need to shorten the catabolic phase related to surgical stress and to achieve this by minimizing insulin resistance in the post-operative period.

\section{Current approaches}

The novel approach adopted has been to challenge the conventional wisdom of fasting prior to surgical procedures. The traditional basis for fasting had been to prevent aspiration, but this view was first challenged in the 1980s and, as a result, many patients were allowed clear fluids up until two hours prior to surgery. The development of low osmolality carbohydrate drinks during the mid 1990s opened up the opportunity of providing surgical patients with a carbohydrate load two to three hours prior to anaesthesia. In a comparative study of 14 patients undergoing colorectal surgery Nygren et al. halved post-operative insulin resistance by the use of such oral therapy. ${ }^{15}$ In a metaanalysis of three small prospective studies, Ljungqvist's team showed that such an approach can lead to a reduction in hospital stay by about 20\%.16 However, a randomized study in Toronto of 26 patients undergoing coronary artery bypass grafting and 12 patients having spinal surgery who received oral carbohydrate supplements in the evening and two hours before surgery showed no improvement in post-operative insulin sensitivity. The patients who received the carbohydrate supplements did, however, have lower blood glucose and higher insulin levels than the traditionally fasted patients, although these differences were not significant. ${ }^{17}$ In contrast, in a randomized study from Brazil of gastrointestinal surgery, the addition of whey protein to the carbohydrate drink appeared to have some added value in terms of reduced inflammatory response and lesser insulin resistance. ${ }^{18}$

The benefits of the ERAS approach to colorectal surgery have been confirmed in several reports. ${ }^{19,20,21}$ It can extend for a significant period after surgery. ${ }^{22}$ The role of pre-operative carbohydrate supplementation has been assessed in a number of trials. The mechanism by which it acts includes a reduction in catabolism of fat and protein. This was shown in a study of 40 patients who underwent an elective laparoscopic colectomy. ${ }^{23}$ However, most studies on the role of pre-operative carbohydrate supplementation have excluded diabetic patients. Clearly the concept of giving a patient with inherent insulin resistance a carbohydrate load prior to surgery is seen by many as "a step too far". Indeed, in 2011 the Joint British Diabetes Societies guideline on the management of adults with diabetes undergoing surgery stated:
"The Enhanced Recovery Partnership Programme recommends the administration of high carbohydrate drinks prior to surgery. This may compromise blood glucose control and is not recommended for people with insulin treated diabetes." 24

However, such a blanket recommendation against the use of high carbohydrate drinks prior to surgery requires re-evaluation. Concerns about potential delays in gastric emptying amongst patients with diabetes and, therefore, the reintroduction of the risk of aspiration, appear unfounded. In a study of 25 patients with type 2 diabetes Gustaffson et al. found no delay in gastric emptying compared to healthy volunteers amongst patients given a carbohydrate drink. ${ }^{25}$ In 2011, in a study from Poland, Jodlowski et al. investigated the benefit of such drinks in 48 patients who were to undergo elective colorectal surgery. Seven had type 2 diabetes. Their conclusion was that a pre-operative oral carbohydrate load was safe and well tolerated by them, although realistically this is much too small a sample from which to make an inference. Overall for both diabetic and non-diabetic patients it improved peri-operative well-being, reduced hunger and reduced insulin blood levels and insulin resistance on day 2 after surgery. ${ }^{26}$ This study is of particular importance against the background of a report from Michigan which investigated risk factors for anastamotic leak following a colectomy amongst 5123 patients. ${ }^{27}$ The occurrence of a leak was not directly associated with the presence of diabetes but, when it did happen, patients with diabetes had a four-fold mortality of $26.3 \%$ compared with $6 \%$ for patients without diabetes. Other evidence would suggest that the use of pre-operative carbohydrate loading might reduce this difference in mortality subsequent to an anastamotic leak. ${ }^{20,21}$ Unfortunately none of these studies included any reference to whether these patients' diabetes was well controlled or whether they had complications of their diabetes.

The importance of insulin resistance as an independent factor associated with prolonged hospital stay cannot be over emphasised. The potential benefits of carbohydrate loading, for patients with diabetes have been shown in the field of cardiac surgery. A randomized double-blind placebo controlled trial in Germany amongst 160 patients undergoing elective cardiac surgery, including 31 with non-insulin treated type 2 diabetes, reported that blood glucose levels, gastric fluid volume and insulin requirement did not differ between groups. However, patients receiving a carbohydrate load required less intra-operative inotropic support after initiation of cardio-pulmonary bypass weaning $(p<0.05) .{ }^{28}$ This study provided no details on how well controlled the patients were or the presence of complications, but did specifically exclude patients with type 1 diabetes after initial recruitment.

\section{Conclusions}

Although patients with diabetes have been excluded from most trials looking at the role of carbohydrate loading as part of an ERAS program, there is certainly a need to stimulate a call for a properly conducted large-scale randomized controlled trial of its efficacy in this group of patients. There is some evidence, albeit 
from small studies, to suggest that carbohydrate loading preoperatively can be safe in patients with diabetes. Naturally there will be a need to carefully monitor and support such patients but this is integral to the ERAS approach. Clearly, its potential to shorten hospital stay, give a more rapid return to normal activities and reduce the frequency of serious complications would be of particular benefit.

\section{Conflict of interest None. Funding None.}

\section{References}

1. Scott NB. Enhanced recovery after surgery - the way forward. Reg Anesth Pain Med 2012;37(Suppl 1):E128-E131

2. Gustafsson UO, Hausel J, Thorell A et al. Adherence to the enhanced recovery after surgery protocol and outcomes after colorectal cancer surgery. Arch Surg 2011;146:571-77. http://dx.doi.org/10.1001/archsurg.2010.309

3. Van den Berghe G. How does blood glucose control with insulin save lives in intensive care? J Clin Invest 2004;114:1187-95. http://dx.doi.org/10.1172/JCI23506

4. Bernard C. Leçons sur le Diabete et la Glycogenese Animale. Baillere (1877) Paris.

5. Ljungqvist O, Nygren J, Thorell A. Modulation of post-operative insulin resistance by pre-operative carbohydrate loading. In: Clinical Nutrition and Metabolism Symposium on'Endocrine and nutritional modulation of the metabolic response to stress'. Proc Nutr Soc 2002:61:329-35.

6. Thorell A, Nygren J \& Ljungqvist O. Insulin resistance - a marker of surgical stress. Curr Opin Clin Nutr Metab Care 1999;2:69-79. http://dx.doi.org/10.1097/00075197-199901000-00012

7. Zerr K, Furnary A, Grunkemeier G et al. Glucose control lowers the risk of wound infection in diabetics after open heart surgery. Ann Thorac Surg 1997;163:356-61. http://dx.doi.org/10.1016/S0003-4975(96)01044-2

8. Furnary AP, Zerr KJ, Grunkemeier GL, et al. Continuous intravenous insulin infusion reduces the incidence of deep sternal wound infection in diabetic patients after cardiac surgical procedures. Ann Thorac Surg 1999;67:352-62. http://dx.doi.org/10.1016/S0003-4975(99) 00014-4

9. Van der Berghe $G$, Wouters $P$, Weeks $F$ et al. Intensive insulin therapy in critically ill patients. N Engl J Med 2001;345:1359-67. http://dx.doi.org/10.1056/NEJMoa011300

10. NICE-SUGAR Study Investigators, Finfer S, Liu B, Chittock DR et al. Hypoglycemia and risk of death in critically ill patients. $N$ Engl J Med 2012;367:1108-18. http://dx.doi.org/10.1056/NEJMoa1204942

11. Buchleitner AM, Martínez-Alonso M, Hernández M et al. Perioperative glycaemic control for diabetic patients undergoing surgery. Cochrane Database Syst Rev 2012;9:CD007315

12. Lanspa MJ1, Hirshberg EL, Phillips GD et al. Moderate glucose control is associated with increased mortality compared with tight glucose control in critically ill patients without diabetes. Chest 2013;143:1226-34. http://dx.doi.org/10.1378/chest.12-2072

13. Okabayashi T, Shima Y, Sumiyoshi T et al. Intensive versus intermediate glucose control in surgical intensive-care unit patients. Diabetes Care 2014; Mar 12. [Epub ahead of print]

14. Signal M, Le Compte A, Shaw GM, et al. Glycemic levels in critically ill patients: are normoglycemia and low variability associated with improved outcomes? J Diabetes Sci Technol 2012;6:1030-7. http://dx.doi.org/10.1177/193229681200600506

15. Nygren J, Soop M, Thorell A et al. Preoperative oral carbohydrate administration reduces postoperative insulin resistance. Clin Nutr 1998;17:65-71. http://dx.doi.org/10.1016/S0261-5614(98)80307-5

16. Ljungqvist $O$, Thorell A, Gutniak M et al. Preoperative nutrition - elective

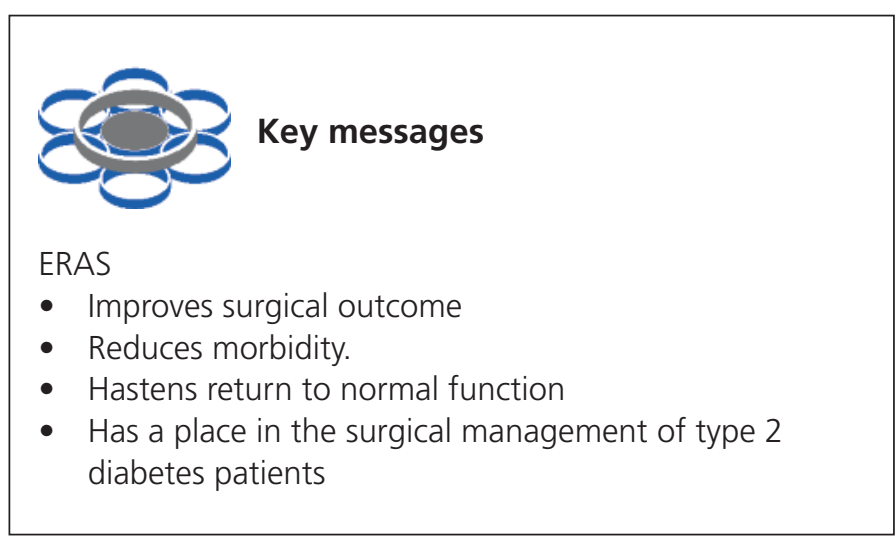

surgery in the fed or the overnight fasted state. Clin Nutr 2001;20(Suppl. 1):167-71. http://dx. doi.org/10.1054/clnu.2001.0462

17. Tran S, Wolever TH, Errett LE et al. Preoperative carbohydrate loading in patients undergoing coronary artery bypass or spinal surgery. Anesth Analg 2013;117:305-13. http://dx.doi.org/10.1213/ ANE.0b013e318295e8d1

18. Perrone F, da-Silva-Filho AC, Adômo IF et al. Effects of preoperative feeding with a whey protein plus carbohydrate drink on the acute phase response and insulin resistance. A randomized trial. Nutr J 2011;10:66-72. http://dx.doi.org/10.1186/1475-2891-10-66

19. Noblett SE, Watson DS, Huong $\mathrm{H}$ et al. Pre-operative oral carbohydrate loading in colorectal surgery: a randomized controlled trial. Colorectal Dis 2006;8:563-9. http://dx.doi.org/10.1111/j.1463-1318. 2006.00965.x

20. Gustafsson UO, Hausel J, Thorell A et al. Adherence to the enhanced recovery after surgery protocol and outcomes after colorectal cancer surgery. Arch Surg 2011;146:571-7. http://dx.doi.org/10.1001/archsurg.2010.309

21. Eskicioglu C, Forbes SS, Aarts MA et al. Enhanced recovery after surgery (ERAS) programs for patients having colorectal surgery: a meta-analysis of randomized trials. J Gastrointest Surg 2009;13:2321-9. http://dx.doi.org/10.1007/s11605-009-0927-2

22. Zargar-Shostani K, Paddison JS, Booth RJ, et al. A prospective study on the influence of a fast-track program on postoperative fatigue and functional recpvery after major colonic surgery. J Surg Res 2009;154:330-5. http://dx.doi.org/10.1016/j.jss.2008.06.023

23. Kanamori R, Shimamura M, Kinoshita M et al. Preoperative carbohydrate administration prevents catabolism of fat and protein in patients undergoing elective laparoscopic colectomy. Final report 1AP5-10. Eur J Anaesthesiol 2012:29:17.

24. Dhatariya K, Flanagan D, Hilton L et al. Management of adults with diabetes undergoing surgery and elective procedures: improving standards (2011). www. diabetes.nhs.uk

25. Gustaffson UO, Nygren J, Thorell A et al. Pre-operative carbohydrate loading may be used in type 2 diabetes patients. Acta Anaesthesiol Scand 2008;52:946-51. http://dx.doi.org/10.1111/j.1399-6576.2008.01599.x

26. Jodlowski T, Dobosz M, Noga M. Preoperative oral carbohydrate load in colorectal surgery reduces insulin resistance and may improve outcomes - preliminary results of prospective randomized study. Clin Nutr 2011;6(Supplements PP052):134.

27. Ziegler MA, Catto JA, Riggs TW et al. Risk factors for anastomotic leak and mortality in diabetic patients undergoing colectomy: analysis from a statewide surgical quality collaborative. Arch Surg 2012;147:600-05. http://dx.doi.org/10.1001/archsurg.2012.77

28. Breuer JP, von Dossow V, von Heymann C et al. Preoperative oral carbohydrate administration to ASA III-IV patients undergoing elective cardiac surgery. Anesth Analg 2006;103:1099-108. http://dx.doi.org/10.1213/01.ane.0000237415.18715.1d 